\title{
An Improved Direction-of-Arrival Estimation via Phase Information of Sparse Solution
}

\author{
Xiansheng Guo ${ }^{1,2}$, Qun Wan ${ }^{2}$, Chunqi Chang ${ }^{1}$, Edmund Y. Lam ${ }^{1}$ \\ ${ }^{1}$ Department of Electrical and Electronic Engineering \\ University of Hong Kong, Pokfulam Road, Hong Kong \\ ${ }^{2}$ Department of Electronic Engineering, \\ University of Electronic Science and Technology of China, Chengdu 610054, PR China \\ Email: \{xsguo,cqchang,elam\}@eee.hku.hk
}

\begin{abstract}
An improved direction-of-arrivals (DOAs) estimation via phase information of sparse solution is presented in this paper. Unlike the conventional sparse source localization approach using the amplitude of sparse solutions only, through a special partition of the receiving data of the sensors, the phase information of the available sparse solutions is also extracted to estimate DOAs. For the true DOAs exactly on the grids which are used to generate the over-complete dictionary, the performance of our method is close to the conventional sparse source localization method. For the true DOAs that are not on the grids, our method is far superior to the conventional method, as demonstrated by several simulation results.
\end{abstract}

\section{INTRODUCTION}

The problem of source localization plays a fundamental role in many fields, such as radar, sonar, acoustic, and seismic sensing. The conventional subspace-type DOAs estimation methods, including MUSIC (MUltiple Slgnal Classification) [1] and ESPRIT (Estimation of Signal Parameters via Rotational Invariance Techniques) [2], need the assumptions that the number of snapshots is sufficient, signal-tonoise ratio (SNR) is moderately high, and the sources are not strongly correlated. Compared with the conventional subspace-type DOAs estimation method, source localization from sparse solution is another effective DOAs estimation method, which can achieve super-resolution without the need for a good initialization or a large number of snapshots, and with lower sensitivity to SNR and to correlation of the sources [3], [4], [5].

However, most of the existing sparse source localization methods only use the amplitudes of the sparse solutions to realize DOAs estimation. For the single measurement vector (SMV) case, the disadvantage is not significant, but for the multiple measurement vector (MMV) case, more information of the sparse solution will be lost.

In order to improve the precision of DOAs estimation further, we propose an improved DOAs estimation approach via phase information of the sparse solution. The main idea is as follows. We partition the receiving data of uniform linear array (ULA) into two or more special parts, then combine them into a new one and use the basis pursuit method to obtain the sparse solutions. The phase information between the sparse solutions is extracted for DOAs estimation. Compared with the existing algorithms, our approach can obtain a satisfactory DOAs estimation whether the true DOAs are on grid or not. Simulation results demonstrate the efficacy of our methods.

\section{DOAs Estimation Via Sparse Solution}

\section{A. The signal model problem}

We consider a ULA of $M$ omnidirectional sensors receiving $K(K<M)$ stationary random signals emanating from far-field point source signals. The element spacing is $d=\lambda / 2$, with $\lambda$ being the signal wavelength, and the DOA of the $k$ th source is denoted by $\theta_{k}$. The received vector of sensors can be expressed as

$$
\mathbf{y}=\mathbf{A}(\theta) \mathbf{s}+\mathbf{w}
$$

where $\mathbf{A}(\theta)=\left[\mathbf{a}\left(\theta_{1}\right), \cdots, \mathbf{a}\left(\theta_{K}\right)\right]$ is a steering matrix with $\mathbf{a}\left(\theta_{k}\right)=\left[1, e^{j \pi \sin \theta_{k}}, \cdots, e^{j \pi(M-1) \sin \theta_{k}}\right]^{T}$ being a steering vector. In addition, $\mathbf{s}=\left[s_{1}, s_{2}, \cdots, s_{K}\right]^{T}$ is a signal vector with $s_{i}(i=1,2, \cdots, K)$ being a far-field point source signal and $\mathrm{w}$ is an unknown noise vector. The problem here is to estimate DOAs $\theta$ from a single measurement $\mathbf{y}$.

\section{B. Amplitude of sparse solution: Conventional sparse source localization method}

In order to cast the above problem into a sparse representation framework, Malioutov et al. [3] defined an overcomplete dictionary $\mathbf{D}$ in terms of all possible source locations $\left\{\hat{\theta}_{1}, \cdots, \hat{\theta}_{N}\right\},(N \gg K, N \gg M)$, where $N$ is the grid number. Then, one can construct the over-complete dictionary $\mathbf{D}$, where $\mathbf{D}=\left[\mathbf{a}\left(\hat{\theta}_{1}\right), \cdots, \mathbf{a}\left(\hat{\theta}_{N}\right)\right]$. Likewise, the source s can be extended to an $N \times 1$ vector $\mathbf{h}$, where the $n$th element $\mathbf{h}(n)$ is nonzero and equal to $s_{k}$ if the source $k$ comes from $\hat{\theta}_{n}$ for some $k$, and zero otherwise.

For the SMV case, the problem (1) is reduced to

$$
\mathbf{y}=\mathbf{D h}+\mathbf{w}
$$

The sparsest representation is the solution to the following optimization problem [6], [7]:

$$
\hat{\mathbf{h}}=\arg \min _{\mathbf{h}}\|\mathbf{y}-\mathbf{D h}\|_{2}^{2}+\lambda\|\mathbf{h}\|_{1},
$$


where the $\ell_{1}$ norm $\|\cdot\|_{1}$ leads to a sparse solution. $\lambda$ is a regularization parameter. The method to choose an appropriate value for $\lambda$ can be found in reference such as [6].

For the MMV case $(L>1)$, the following optimization can be used:

$$
\hat{\mathbf{H}}=\min _{\mathbf{H}}\|\mathbf{Y}-\mathbf{D H}\|_{\mathrm{F}}^{2}+\lambda\left\|\sum_{j=1}^{L} \mathbf{H}(i, j)\right\|_{1},
$$

where the $\|\cdot\|_{F}$ is the Frobenius norm, $\mathbf{Y}=\left[\mathbf{y}_{1}, \cdots, \mathbf{y}_{L}\right]$ and $\mathbf{H}=\left[\mathbf{h}_{1}, \cdots, \mathbf{h}_{L}\right]$ with $L$ bing the number of measurement. Most of the existing DOAs estimation methods use the amplitude of sparse solutions to realize sources localization, i.e., the indices of the non-zero row of $\hat{\mathbf{H}}$ will give the DOAs estimation. For the sake of comparison, we denote the DOAs estimation via the indices of non-zero row as $\hat{\theta}^{\text {ind }}$.

\section{Phase of sparse solution: An improved sparse source localization method}

We define two permutation matrices $\mathbf{J}_{1}$ and $\mathbf{J}_{2}$ as $\mathbf{J}_{1}=$ $\left[\begin{array}{ll}\mathbf{I}_{M-1} & \mathbf{0}\end{array}\right]_{(M-1) \times M}$ and $\mathbf{J}_{2}=\left[\begin{array}{ll}\mathbf{0} & \mathbf{I}_{M-1}\end{array}\right]_{(M-1) \times M}$ respectively, where $\mathbf{I}_{M-1}$ is a $(M-1) \times(M-1)$ identity matrix. By premultiplication of permutation matrices, one can obtain two different parts of (2) as follows:

$$
\begin{aligned}
& \mathbf{y}_{1}=\mathbf{J}_{1} \mathbf{y}=\mathbf{J}_{1} \mathbf{D h}+\mathbf{J}_{1} \mathbf{w}=\mathbf{D}_{1} \mathbf{h}+\mathbf{w}_{1} \\
& \mathbf{y}_{2}=\mathbf{J}_{2} \mathbf{y}=\mathbf{J}_{2} \mathbf{D h}+\mathbf{J}_{2} \mathbf{w}=\mathbf{D}_{2} \mathbf{h}+\mathbf{w}_{2}
\end{aligned}
$$

where $\mathbf{y}_{1}=\left[y_{1}, \cdots, y_{M-1}\right]^{T}, \mathbf{y}_{2}=\left[y_{2}, \cdots, y_{M}\right]^{T}$, and the others have similar forms. Obviously, $\mathbf{D}_{1}$ and $\mathbf{D}_{2}$ have the special relationship as

$$
\mathbf{D}_{2}=\mathbf{D}_{1} \boldsymbol{\Phi}
$$

in which $\boldsymbol{\Phi}=\operatorname{diag}\left(\left[e^{j \pi \sin \hat{\theta}_{1}}, \cdots, e^{j \pi \sin \hat{\theta}_{N}}\right]\right)$. In the following, we can combine equations (5) and (6) into one:

$$
\begin{aligned}
\mathbf{Y} & =\left[\mathbf{y}_{1}, \mathbf{y}_{2}\right]=\left[\mathbf{D}_{1}, \mathbf{D}_{2}\right] \mathbf{h}+\left[\mathbf{w}_{1}, \mathbf{w}_{2}\right] \\
& =\mathbf{D}_{1}[\mathbf{I}, \boldsymbol{\Phi}] \mathbf{h}+\left[\mathbf{w}_{1}, \mathbf{w}_{2}\right] \\
& =\mathbf{D}_{1} \mathbf{H}+\mathbf{W},
\end{aligned}
$$

where $\mathbf{H}=[\mathbf{I}, \boldsymbol{\Phi}] \mathbf{h}=\left[\mathbf{h}_{1}, \mathbf{h}_{2}\right] \in C^{N \times 2}$. The sparse solution of (8) can be given by convex optimization of (4), which can be solved efficiently in a second-order cone programming (SOCP) framework[8].

From the above partitioning of receiving data, we find that the phase information between sparse solutions also can be extracted to DOAs estimation, similar to the amplitude of sparse solution. In order to discover the sparse solution information, we depict the structure of sparse solution $\hat{\mathbf{H}}$ in Table I when the number of sources $K$ equals to two. From the figure, the nonzero entries in the same row of sparse solutions are related as

$$
h_{2 i}=e^{j \pi \sin \theta_{i}} h_{1 i}
$$

and

$$
\hat{\theta}_{i}^{\text {pha }}=\sin ^{-1}\left[\operatorname{angle}\left(h_{2 i} / h_{1 i}\right) / \pi\right]
$$

where $\hat{\theta}^{\text {pha }}$ denotes the DOAs estimation via the phase of the sparse solution, and angle $(\cdot)$ denotes the phase operator.

TABLE I

THE STRUCTURE OF SPARSE SOLUTION WHEN THE SOURCE NUMBER $K=2$ AND THE MEASUREMENT NUMBER $L=2$, WHERE “..." DENOTES ZERO, AND THE OTHERS ARE NONZERO ENTRIES.

\begin{tabular}{|c|c|}
\hline \multicolumn{2}{|c|}{$\mathbf{H}$} \\
\hline $\mathbf{h}_{\mathbf{1}}$ & $\mathbf{h}_{\mathbf{2}}$ \\
\hline$\ldots$ & $\ldots$ \\
\hline$h_{1 i}$ & $h_{1 j}$ \\
\hline$\ldots$ & $\cdots$ \\
\hline$h_{2 i}$ & $h_{2 j}$ \\
\hline$\ldots$ & $\ldots$ \\
\hline
\end{tabular}

\section{Discussion}

For our method there are other aspects to be noted:

1) Although our method considers the $L=2$ case, it can be easily generalized to $L>2$, which will generate more phase information to be used. However, too big a value of $L$ will degenerate the performance of DOAs estimation, hence a reasonable $L$ may be $M-K>L \geq 2$.

2) If a refining grid strategy is used, the performance of our method will be close to the conventional method, and so how to combine the two results is worthy of further study.

3) The proposed method considers the ULA case. In fact, it can extend to other manifolds which satisfy the rotational invariance property (RIP), such as uniform circular array (UCA), uniform plane array, etc.

4) The performance of the amplitude-based method is worse than the phase-based method because the former only uses the indices of the nonzero row of sparse solutions. Therefore, the information is limited for both SMV and MMV, while the phase-based method can use more information between sparse solutions for the MMV case. With more measurements, we can have more precise DOA estimation results. This also demonstrates that MMV is superior to SMV in many cases [9], [10].

\section{Simulation RESUltS}

To demonstrate the efficacy of the proposed method, three simulation results are presented here. The performance measure used in this paper involves the cumulative DOAs estimation error, which is defined as $\sum_{i=1}^{I}\left|\hat{\theta}_{i}-\theta\right|$, where $I$ is the number of independent trials, $\hat{\theta}_{i}$ is the $i$ th DOA estimation, and $\theta$ is the true DOA. 


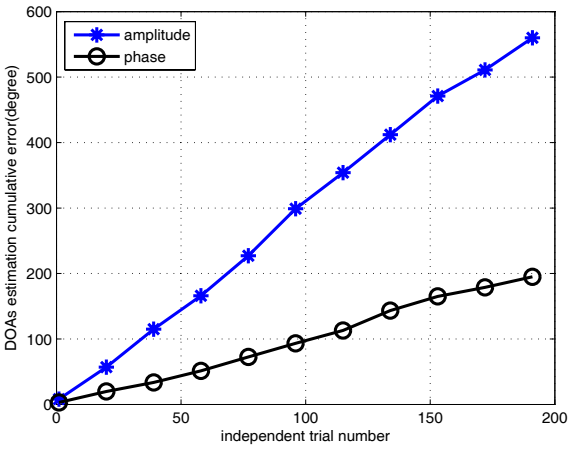

(a) $M=8$, SNR=10dB, $\theta=50^{\circ}$.

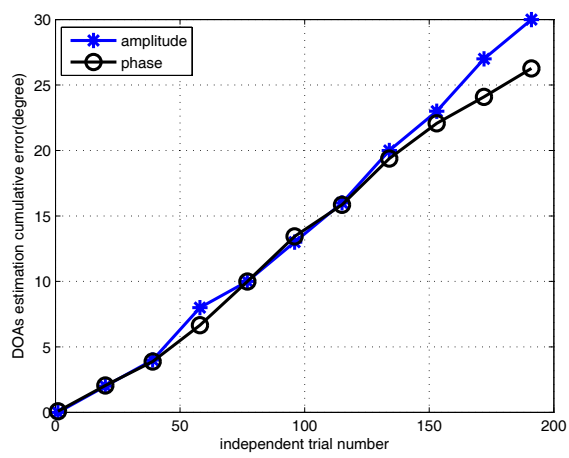

(b) $M=8$, SNR=30dB, $\theta=50^{\circ}$.

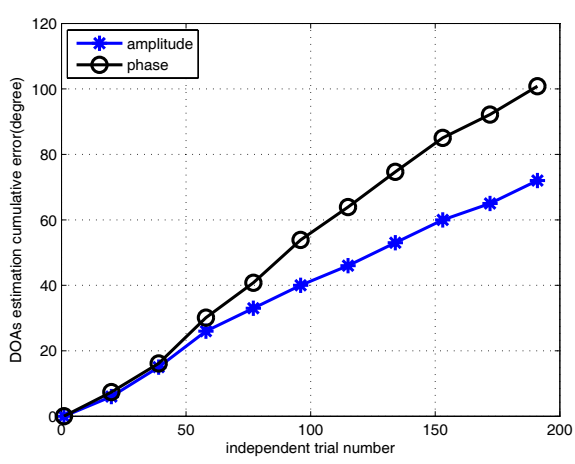

(c) $M=16$, SNR=10dB, $\theta=50^{\circ}$.

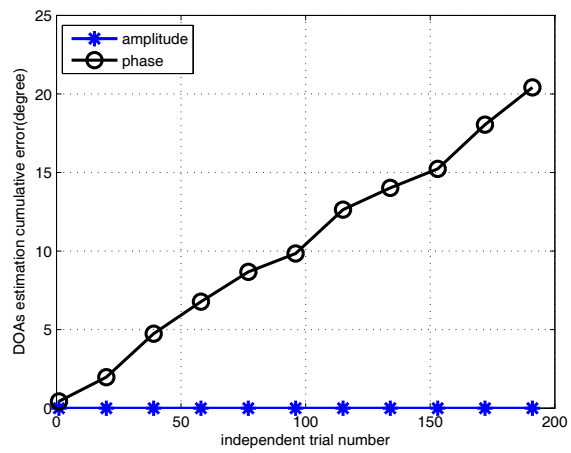

(d) $M=16$, SNR=30dB, $\theta=50^{\circ}$.

Fig. 1. The DOAs cumulative (absolute) errors for different cases.
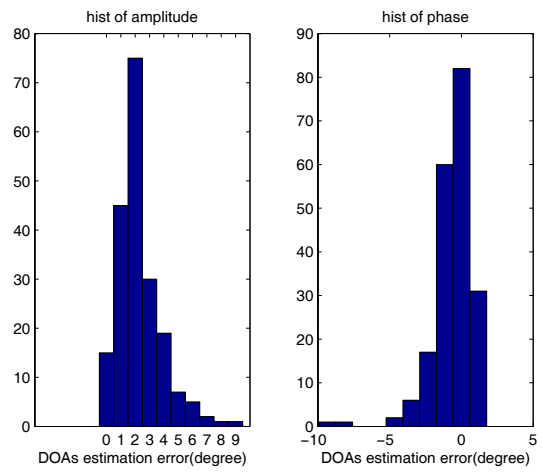

(a) $M=8$, SNR=10dB, $\theta=50.5^{\circ}$.
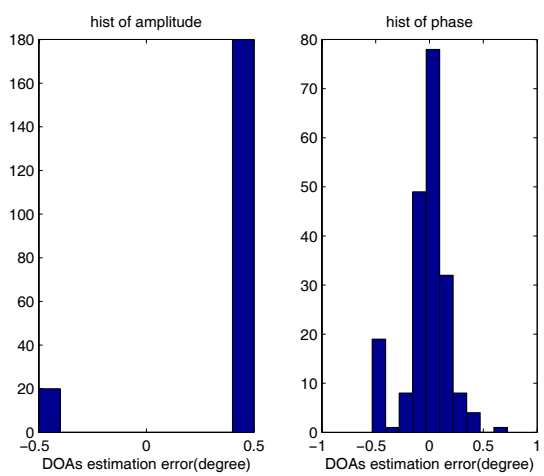

(b) $M=8$, SNR=30dB, $\theta=50.5^{\circ}$.
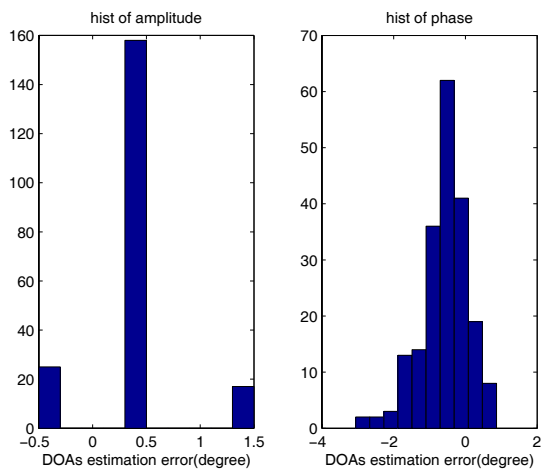

(c) $M=16, \mathrm{SNR}=10 \mathrm{~dB}, \theta=50.5^{\circ}$.
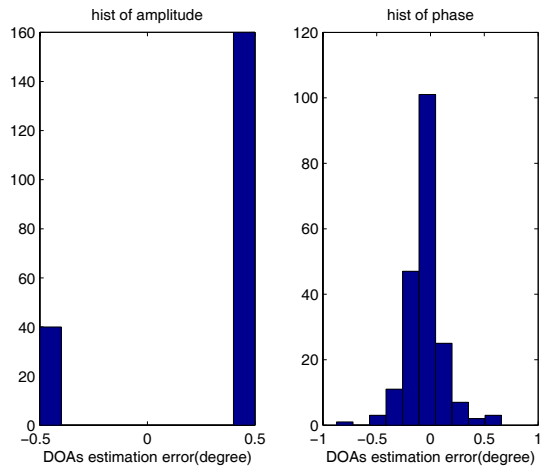

(d) $M=16, \mathrm{SNR}=30 \mathrm{~dB}, \theta=50.5^{\circ}$.

Fig. 2. The histogram of DOAs estimation errors for different cases. 


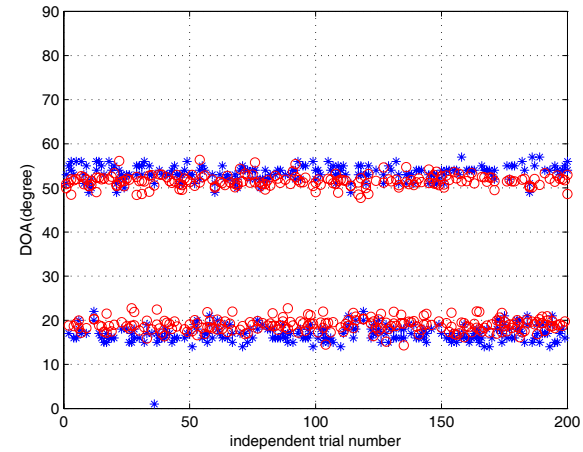

Fig. 3. Two DOAs estimation results, with sensor number $M=12$, SNR $=30 \mathrm{~dB}$, and the true DOA $\theta_{1}=20^{\circ}, \theta_{2}=50.5^{\circ}$.

\section{A. One source, true $D O A$ is on the grid}

The true DOA is from $50^{\circ}$. The possible source DOAs are confined from $1^{\circ}$ to $89^{\circ}$ and the distance between adjacent grids is $1^{\circ}$, so the grid number is $N=89$. An additive white Gaussian noise is added. The signal-to-noise ratio (SNR) is $10 \mathrm{~dB}$ and $30 \mathrm{~dB}$, respectively, and the sensor number is 8 and 16, respectively. The convex optimization of equation (8) is solved by SPGL1 [11]. The cumulative DOAs estimation error against 200 independent trials are shown in Fig. 1.

From the figure, we find that the DOAs estimation from $\hat{\theta}^{\text {pha }}$ is more precise than $\hat{\theta}^{\text {ind }}$ when SNR and $M$ are lower (Figs. 1 (a) and (b)). Note that for high SNR and $M$, the former is slightly worse than the latter, but the difference is not remarkable. The mean bias of Fig. 1 (c) is less than $0.2^{\circ}$, while the mean bias of Fig. 1 (d) is no more than $0.1^{\circ}$. For higher SNR and $M$, the DOAs estimation precision of the former is higher than the latter mainly because the true DOA is on grid. If it is not on grid, the fixed bias will exist whenever $M$ and SNR are lower or higher (see below).

\section{B. One source, true DOA is not on the grid}

All the simulation conditions are the same with the example above except that the true DOA is from $50.5^{\circ}$. For different $M$ and SNR cases, the histogram of DOAs estimation errors with 200 independent trials are shown in Fig. 2.

For the case that the true DOA is not on the grid, the phase-based method is superior to the amplitude-based method for different SNR and $M$ cases. All histogram figures show that $\hat{\theta}^{\text {ind }}$ has a fixed bias for different cases because of the grid problem, i.e., $\hat{\theta}^{\text {ind }}$ is seriously influenced by the grid distance. Although a refining grid strategy [3] can improve the estimation results, it will also increase the computational burden. In addition, the refining grid strategy cannot solve the DOAs estimation precision problem fundamentally.

\section{Two sources, one of them is on the grid, the other is not}

Let us consider an example with sensor number $M=12$, the source number $K=2$, the true DOAs $\theta_{1}=20^{\circ}$ and $\theta_{2}=50.5^{\circ}$, and SNR $=30 \mathrm{~dB}$. All possible DOAs locations are from $1^{\circ}$ to $90^{\circ}$. The grid to generate an over-complete dictionary is $1^{\circ}$, so the grid number is $N=90$. The DOAs estimation is run with 200 independent trials. The DOAs estimations using amplitude information (blue $*$ ) and phase information (red o) are depicted in Fig. 3.

From the figure, we find that the phase-based method can improve the DOAs estimation results efficiently when the source number $K$ equals to two. When the true DOA is on grid $\left(\theta_{1}=20^{\circ}\right)$, the performance of amplitude-based method is close to the phase-based method, while for the case that true DOA is not on grid $\left(\theta_{2}=50.5^{\circ}\right)$, the amplitude-based method is much worse than the phasebased method.

\section{CONCLUSION}

In this paper, we propose an improved DOAs estimation method via phase information of the sparse solution. The existing methods use only the amplitude information (indices of nonzero entries) of sparse solutions to determine the source locations. Through a special partitioning of receiving data, the phase information is extracted from the sparse solution to give more precise DOAs estimation. Simulation results proved the efficacy of the proposed method.

\section{ACKNOWLEDGMENT}

This work was supported by a grant from the National Natural Science Foundation of China (No.60772146), the National High Technology Research and Development Program of China (863 Program, No.2008AA12Z306), and the University of Hong Kong (Project 10208648).

\section{REFERENCES}

[1] R. Schmidt, "Multiple emitter location and signal parameter estimation," IEEE Trans. Antennas Propagat., vol. 34, pp. 276-280, 1986.

[2] R. Roy, A. Paulraj, and T. Kailath, "ESPRIT- a subspace rotation approach to estimation of parameters of cisoids in noise," IEEE Trans. Acoust., Speech, Signal Processing, vol. 34, pp. 1340-1342, 1986.

[3] D. Malioutov, M. Çetin, and A. S. Willsky, "A sparse signal reconstruction perspective for source localization with sensor arrays," IEEE Trans. Signal Processing, vol. 53, pp. 3010-3022, 2005.

[4] A. Ebeck, P. Stoica, and J. Li, "Exact and approximate solutions of source localization problems," IEEE Trans. Signal Processing, vol. 56, pp. 1770-1778, 2008.

[5] S. Maria and J. J. Fuchs, "Application of the global matched filter to stap data: An efficient algorithmic approach," in Proc. ICASSP, Toulouse, France, May 2006, pp. IV 1013-IV 1016.

[6] I. F. Gorodnitsky and B. D. Rao, "Sparse signal reconstructions from limited data using FOCUSS: A re-weighted minimum norm algorithm," IEEE Trans. Signal Processing, vol. 45, pp. 600-616, 1997.

[7] B. D. Jeffs, "Sparse inverse solution methods for signal and image processing applications," in Proc. ICASSP, Seattle, WA(USA), May 1998, pp. $1885-1888$.

[8] M. Lobo, L. Vandenberghe, and S. Boyd. (2008, Dec.) SOCP: Software for second-order cone programming. [Online]. Available: http://www.stanford.edu/ boyd/old-software/SOCP

[9] B. D. Rao, K. Egan, and S. Cotter, "Diversity measure minimization based method for computer sparse solutions to linear inverse problem with multiple measurement," in Proc. ICASSP, Montreal Canada, May 2004, pp. II 369-II 372.

[10] J. Chen and X. Huo, "Theoretical results on sparse representations of multiple-measurement vectors," IEEE Trans. Signal Processing, vol. 54, pp. 4634-4643, 2006.

[11] E. V. Berg and M. P. Friedlander. (2007, June) The SPGL1 package. [Online]. Available: http://www.cs.ubc.ca/labs/scl/spgl1 